\title{
Anti-VEGF/ANG2 Nanobody BI 836880
}

National Cancer Institute

\section{Source}

National Cancer Institute. Anti-VEGF/ANG2 Nanobody BI 836880. NCI Thesaurus. Code C126644.

A nanobody directed ag ainst angiopoietin-2 (Ang2; ANGPT2)- and vascular endothelial growth factor (VEGF)-derived peptides, with potential antiang iog enic and antineoplastic activities. Anti-VEGF/ANG2 nanobody BI 836880 binds to Ang 2 and VEGF and inhibits receptor binding; this prevents Ang2- and VEGF-mediated signaling and inhibits both tumor angiogenesis and tumor cell proliferation. Both VEGF and Ang2 are upregulated in a variety of cancer cell types and play a crucial role in angiogenesis. The nanobody is based on functional fragments of sing le-chain antibodies. 